\title{
On Coordinating Time Series Spectroscopy with the WET
}

\author{
S. E. Thompson ${ }^{1}$ \\ ${ }^{1}$ Delaware Asteroseismic Research Center, Mt. Cuba Observatory and University of \\ Delaware, Dept. of Physics and Astronomy, Newark, DE 19716, USA
}

\begin{abstract}
Recently time series spectroscopy of hydrogen atmosphere white dwarfs (DAVs) has proven successful in measuring the spherical degree and pulsation velocity of each mode. The information gained from these observations would be enhanced by having the observations coincide with a multi-site photometry, such as that provided by a Whole Earth Telescope (WET). The accurate photometry would provide the precise periods and photometric amplitudes that cannot be easily obtained by spectroscopy alone. Combining spectroscopy with photometry of DAVs will lead to answers regarding combination modes, convective driving, and the pulsation geometry of each mode. Here I discuss how time series spectroscopy will improve the results of the photometric runs and the quality and quantity needed for success.
\end{abstract}

\section{Introduction}

The Whole Earth Telescope (WET) is an ideal tool for accurately identifying and measuring the pulsation periods of variable stars. A common target of these multi-site campaigns has been hydrogen atmosphere white dwarf pulsators (DAVs). The scientific goals for studying DAVs have included modeling white dwarf (WD) interiors (e.g. Clemens 1993), measuring convection from light curve shapes (Montgomery 2005, 2007), and measuring cooling rates from period changes (e.g. Kepler et al. 1991). The ultimate success of these projects depend, in part, on knowing the spherical degree, $\ell$. However since the photometry of the WET does not directly measure $\ell$, we must either infer the values from asteroseismic models, or rely on other methods. 
Time series spectroscopy (TSS) of WDs has proven successful for measuring $\ell$ and pulsation velocity. The technique depends on subtle variations in Hydrogen line to distinguish between different values of $\ell$ and small shifts in the spectral line to measure the velocity. The required high signal-to-noise and short exposure times limit the observations to some of the largest operating telescopes. As such, obtaining long, continuous spectroscopic sets is almost impossible, resulting in poorly measured periods and no identification of closely spaced modes. However, this short-coming can be surmounted by taking the spectra during a WET run and using the multi-site photometry to identify the pulsation periods.

\section{Measurements with TSS}

By taking TSS of DAVs we can measure aspects of pulsation that time series photometry cannot. First, Doppler shifts of the spectral lines measure the longitudinal motion of the pulsation, perpendicular to our line of sight. This motion causes the observed brightness variations and thus is a more fundamental measure of the pulsation amplitude. Observing velocities allows us to distinguish those flux variations associated with physical pulsation from those created by interacting with the convection zone (van Kerkwijk et al. 2000). Second, the Hydrogen lines change shape along with the stellar flux variations in accordance to the distribution of the pulsation across the stellar surface. Since limb darkening varies across the spectral lines, each wavelength samples a different area of stellar surface. Each spherical degree, with their unique latitudinal distribution, shows a different amount of cancellation when integrated over the observed surface area. Since the total surface area varies with limb darkening, the amplitude of the pulsation at each wavelength will be unique to that value of $\ell$ (Robinson et al. 1995).

The most successful TSS white dwarf target is G29-38. As the brightest large amplitude DAV, observations from Keck and the VLT have identified $\ell$ for 12 different modes from the Hydrogen line shapes (Clemens et al. 2000, Kotak et al. 2002, Thompson et al. 2006). Additionally, van Kerkwijk et al. (2000) and Thompson et al. (2003) have measured the velocity associated with the largest pulation modes. They show that the ratio of the velocity and the flux amplitudes for periods near $800 \mathrm{~s}$ are consistent with those predicted by the convective driving theory (Wu \& Goldreich 1999).

Part of the success of TSS is exposing new mysteries. Though TSS for mode identification has only been applied successfully to $\approx 16$ modes on four stars, 5 of those modes are not $\ell=1$, leaving the impression that higher $\ell$ modes are more common than previously thought. The ratio of the velocity to flux amplitudes have not been accurately measured for shorter periods, but those 
measurements that do exist indicate that the measurements may not follow the predictions of the convective driving theory (see Thompson et al. 2003). Pulsation velocity measurements of G29-38 have shown that the physical pulsations produce combination velocity modes. Are these velocity combinations unique to G29-38? Are they actually resonant modes, or is there a force causing nonlinear mixing of the physical pulsations of the star?

The results for G29-38 come from runs less than 6 hours, leaving the precision of the measured frequencies to at best $45 \mu \mathrm{Hz}$. Large amplitude pulsators, like G29-38, tend to show a complex set of pulsation modes that vary between observing runs. To know the true pulsation structure of the star, we need longer coverage, but we do not necessarily need more observations if a WET run coincides with the TSS observations. The WET can provide the extended coverage to measure the frequencies of pulsation. If TSS is taken over several nights (e.g. 3 nights provides a frequency resolution of $6 \mu \mathrm{Hz}$ ), the worry of which alias peak to select among the spectroscopic FTs is not a problem, the WET would have already identified and measured the frequencies. In this way extended photometry will improve the output of the TSS while the TSS will provide crucial information about $\ell$ and pulsation physics that ultimately will help decipher the star.

Among the WD pulsators, the DAVs, with their deep hydrogen lines, show the greatest variation between different spherical degree modes. Also, with the abundance of known DAVs (see Castanheira et al. 2006, Mullally et al. 2005, and Mukadam et al. 2004), we are more likely to measure spherical degrees and pulsation velocities on an interesting number of targets.

\section{Measuring spherical degree}

Thompson et al. (2004) introduced a new technique that obtains $\ell$ from data that previously had been of too poor of quality to perform the task. By fitting the spectra with the combination of a Lorentzian and a Gaussian, they focused on only the necessary information contained in the spectra to determine the spherical degree. In this case they simplified the periodic line shape variations by only allowing the height of the continuum along with the areas of the Gaussian and the Lorentzian to change from the initial fit to the average spectrum. When compared to models, it is clear that the measurements do not accurately agree with the observations. However, for G29-38 most modes have the same line variations, and most closely resemble the $\ell=1$ model (Clemens et al. 2000).

As a simple method to demonstrate the relationship between the observations and the models, we measure the Gaussian equivalent width (EW) and the Lorentzian EW from the spectral fits. We then plot the amplitudes of these variations against each other after normalizing by the flux amplitude of 


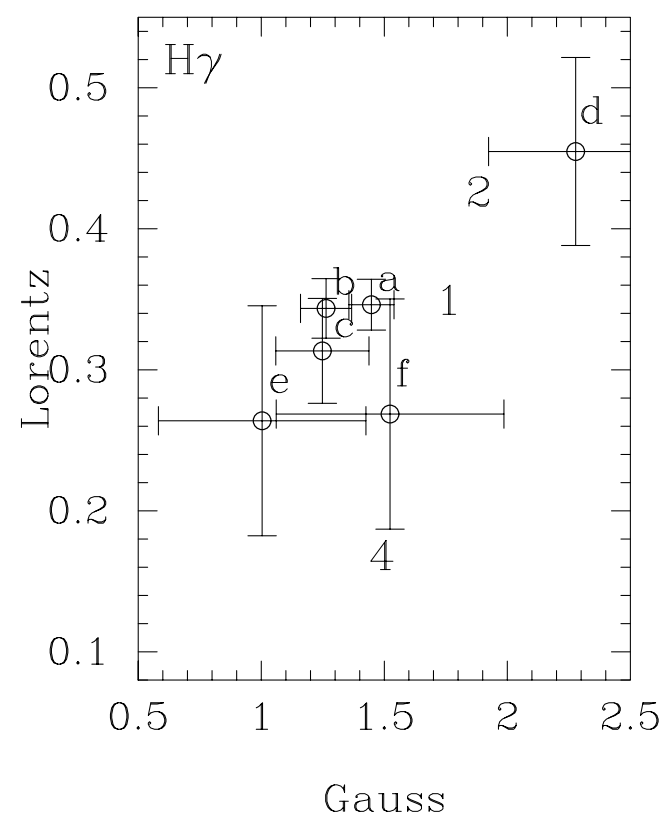

Figure 1: The normalized Gaussian EW vs. the normalized Lorentzian EW amplitudes measured for the Hydrogen-gamma line for pulsated model DAV spectra (numbers), and for observations of G29-38 observed with the Keck telescope (a-f) (Clemens et al. 2000). The different $\ell$ as established with pulsated atmospheric models of DAVs are labelled according to their value. Only the six largest modes were measured in this manner. Most modes cluster near the $\ell=1$ mode, though show a distinct offset from the model. Mode 'a' (614 s) is $\ell=1$ while mode 'd' (776 s) is $\ell=2$.

the mode. Fitting pulsated model spectra of a DAV shows that these amplitudes are different for different $\ell$. See Figure 1 for a comparison of the model to observations of G29-38. The majority of modes are $\ell=1$ while the one $\ell=2$ mode has larger Gaussian and Lorentzian EW amplitudes (Clemens et al. 2000 and Thompson et al. 2006). The models agree with this trend, showing $\ell=2$ with larger Gaussian and Lorentzian EW amplitudes. The $\ell=1$ identification of the $615 \mathrm{~s}$ mode is supported by the convective fitting analysis of Montgomery (2005). The $\ell=2$ measuremnt for the $776 \mathrm{~s}$ mode is supported by the especially large velocity amplitude for this mode (van Kerkwijk et al. 2000).

With this analysis we have a simple way to determine the quality of data needed to distinctly measure $\ell$. The quality of data affects our ability to accurately fit each spectrum and thus the size of our error bars. As long as our error bars are smaller than the distance between the $\ell=1$ and $\ell=2$ models, we are 
likely to make the correct measurement of $\ell$. At this modest resolution, most spectrographs will provide a wide enough wavelength range to allow us to measure more than one spectral line, thus we can obtain 2-3 measurements of $\ell$ for each mode from one set of TSS.

\section{TSS Quantity and Quality}

By running simmulations of noisy model spectra, I determine the quantity and quality of the spectroscopy needed to measure $\ell$ with TSS. Aspects of the measurement that affect the ability to measure $\ell$ include: the signal-to-noise of the individual spectrum, the resolution of the spectrum, the total number of measurements, and the time span these measurements cover. For our example here, let us assume the time span is enough to accurately distinguish all frequencies, and are known accurately from supporting photometry. From previous experience we find that only a modest resolution $(\leq 7 \AA)$ is necessary to measure the changes in line shape. This resolution is usually set by the seeing since a wide slit is used to gather as much light as possible. Getting the required $S / N$ is the tricky part; to reach more than ten DAVs requires observing stars as dim as 16 mag. Eight meter class telescopes can obtain a $\mathrm{S} / \mathrm{N}$ of 70 for a $15.5 \mathrm{mag}$ star with a $30 \mathrm{~s}$ exposure; the cycle time is then near $50 \mathrm{~s}$.

I ran simmulated spectra with a resolution of $6 \AA$ (and a dispersion of $1.8 \AA /$ pixel), $\mathrm{S} / \mathrm{N}$ of 70 , and cycle time of $50 \mathrm{~s}$ to determine the total length of observations necessary to measure various amplitude pulsation modes on a DAV. The expected significance of our $\ell$ measurements can be found in Figure 2 . Since the goal is to uniquely measure $\ell$, we judge significance based on whether an $\ell=2$ could be distinguished from an $\ell=1$ in the $\mathrm{H} \gamma$ line. However, remember it is likely that the same spectra will also measure $\mathrm{H} \beta$ and $\mathrm{H} \delta$. The ordinate in Figure 2, labelled "significance" is the ratio between the measured error bar and the difference between the $\ell=1$ and $\ell=2$ models for the $\mathrm{H} \gamma$ line. To observe the $8 \mathrm{mma}(0.8 \%)$ modes, we would need almost 16 hours of telescope time. However with only 4 hours, we could measure the spherical degrees of the large $20 \mathrm{mma}$ modes.

\section{Measure Velocities}

The same spectra that measures $\ell$ can be used to measure the velocity of each mode. However, this is not the best way to measure velocities. While sacrificing resolution for more light improves our $\ell$ measurements, it deteriorates the velocity measurements. Thompson et al. (2003) showed that with higher resolution spectra $(0.215 \AA, S / N \sim 8,5$ hrs of observations), they could significantly improve the velocity measurements. For the higher resolution data, they 


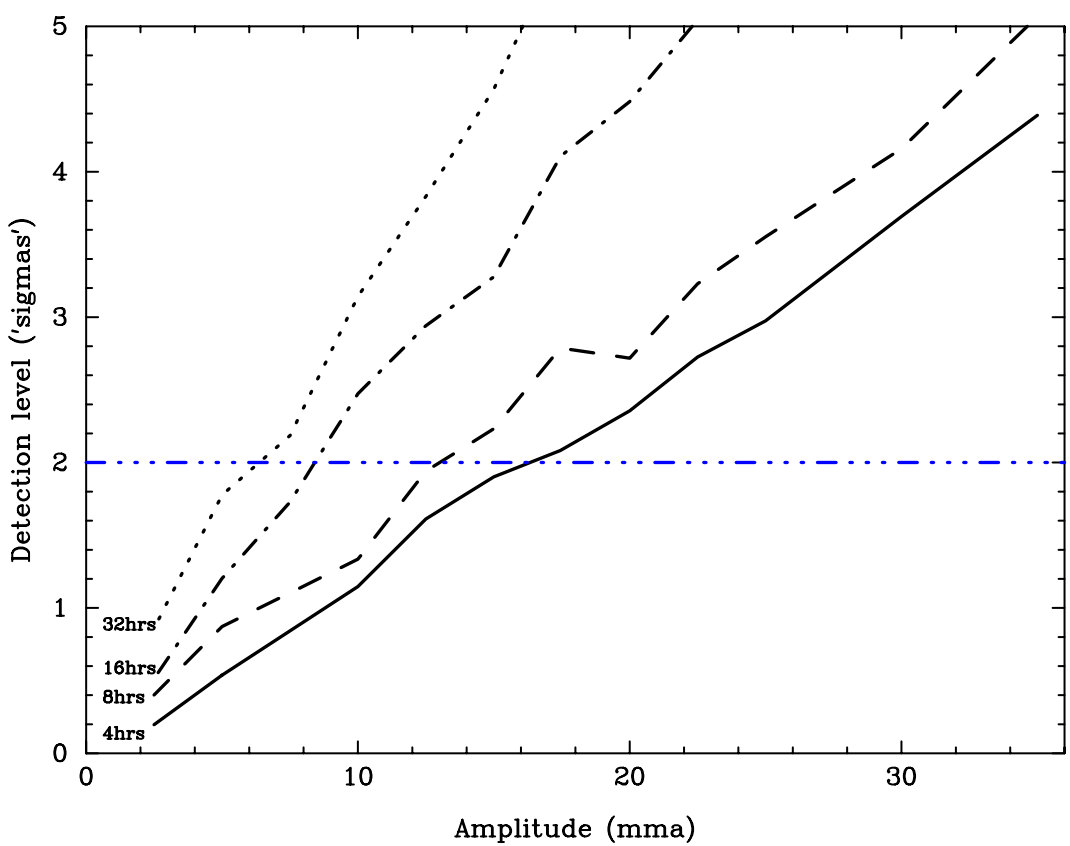

Figure 2: The TSS detection significance to distinguish between $\ell=1$ and $\ell=2$ of different amplitude modes given various length of observing runs. These values come from simulations of noisy $\mathrm{H} \gamma$ spectra with a $\mathrm{S} / \mathrm{N}$ of 70 and an observational cycle time of $50 \mathrm{~s}$. The simmulation assumes the pulsation periods are known either from the spectroscopy or coincidental photometry.

could detect velocity amplitudes down to $1 \mathrm{kms}^{-1}$ while for the lower resolution spectra ( $\sim 7 \AA$, van Kerkwijk et al. 2000) they were limited to those above $2 \mathrm{kms}^{-1}$. The lower noise level revealed velocities for modes with flux amplitudes as small as $10 \mathrm{mma}$. To obtain this higher resolution you must observe with a slit and reduce the spectral range, both significantly reduce the amount of light gathered, and deteriorate the measured flux amplitudes.

By measuring the velocities, we can explore the nature of the combination modes and the validity of the convective driving theory proposed for DAVs. The velocity, or lack thereof, will reveal which modes are physical pulsations on the star and which are combination modes. Combinations generally have shorter periods and lower amplitudes, requring higher quality velocity measurements. The ratio between velocity amplitudes and flux amplitudes seem to agree with observations for the longer periods ( $800 \mathrm{~s}$ ) but have not been adequately tested for the short periods. Unfortunately these short period modes tend to 
have smaller flux and velocity amplitudes. The limiting factor from the previous high resolution spectra was the poor light curve. At least for G29-38, velocity measurements of a significant number of short period, lower amplitude modes is possible, but requires coordination with a set of photometric observations to obtain the flux amplitudes and precise frequencies.

\section{Conclusions}

TSS, though a fairly new tool for DAVs, has proven its effectivness, especially for the DAV G29-38 by measuring velocity pulsations and $\ell$. Both high and low resolution TSS have measured the pulsation velocities of G29-38. In the future, if such studies are combined with photometry, they could provide conclusive tests of the convective driving theory, and further examination of the nature of the combination modes.

The improvements in TSS by fitting spectra and spectrograph efficiency make it possible to perform $\ell$ identification for currently about ten DAVs. Mode identification is generally inferred from the very models that are used to constrain the stellar structure. While blindly choosing $\ell=1$ is a good guess, now that we know of at least three $\ell=2$ and two $\ell \geq 3$ modes (Thompson et al. 2004; Kotak et al. 2002; Thompson et al. 2008) after successful TSS analysis of only 16 modes on DAVs, we must question this guess. If the goal of the WET, or any multi-site photometric study, is to perform asteroseismology of a DAV, I encourage the study to pursue TSS to improve the validity of their mode identifications and ultimately their measurements of stellar structure.

TSS must rely on additional photometry to accurately know the pulsation frequencies, however photometry must rely on spectroscopy to measure the physical pulsation velocity and $\ell$. The coordination of these two observations will improve the information gained from either acting alone.

Acknowledgments. I acknowledge M. H. van Kerkwijk for proving the reduced Keck spectra of G29-38 and the Crystal Trust for their contribution to the completion of this work.

\section{References}

Castanheira, B. G., Kepler, S. O., Mullally, F., et al. 2006, A\&A, 450, 227

Clemens, J. C. 1993, Baltic Astronomy, 2, 407

Clemens, J. C., van Kerkwijk, M. H., \& Wu, Y. 2000, MNRAS, 314, 220

Goldreich, P., \& Wu, Y. 1999, ApJ, 511, 904

Kepler, S. O., Winget, D. E., Nather, R. E., et al. 1991, ApJL, 378, L45 
Kotak, R., van Kerkwijk, M. H., \& Clemens, J. C. 2002, A\&A, 388, 219

Montgomery, M. H. 2005, ApJ, 633, 1142

Montgomery, M. H. 2007, Astronomical Society of the Pacific Conference Series, 372,635

Mukadam, A., Mullally, F., Nather, R. E., et al. 2004, ApJ, 607, 982

Mullally, F., Thompson, S. E., Castanheira, B. G., et al. 2005, ApJ, 625, 966

Robinson, E. L., Mailloux, T. M., Zhang, E., et al. 1995, ApJ, 438, 908

Thompson, S. E.,Clemens, J. C., van Kerkwijk, M. H., \& Koester, D. 2003, ApJ, 589, 921

Thompson, S. E., Clemens, J. C., van Kerkwijk, M. H., et al. 2004, ApJ, 610, 1001

Thompson, S. E, van Kerkwijk, M. H., \& Clemens, J. C. 2008 MNRAS, submitted van Kerkwijk, M. H., Clemens, J. C., \& Wu, Y. 2000, MNRAS, 314, 209

Wu, Y., \& Goldreich, P. 1999, ApJ, 519, 783 


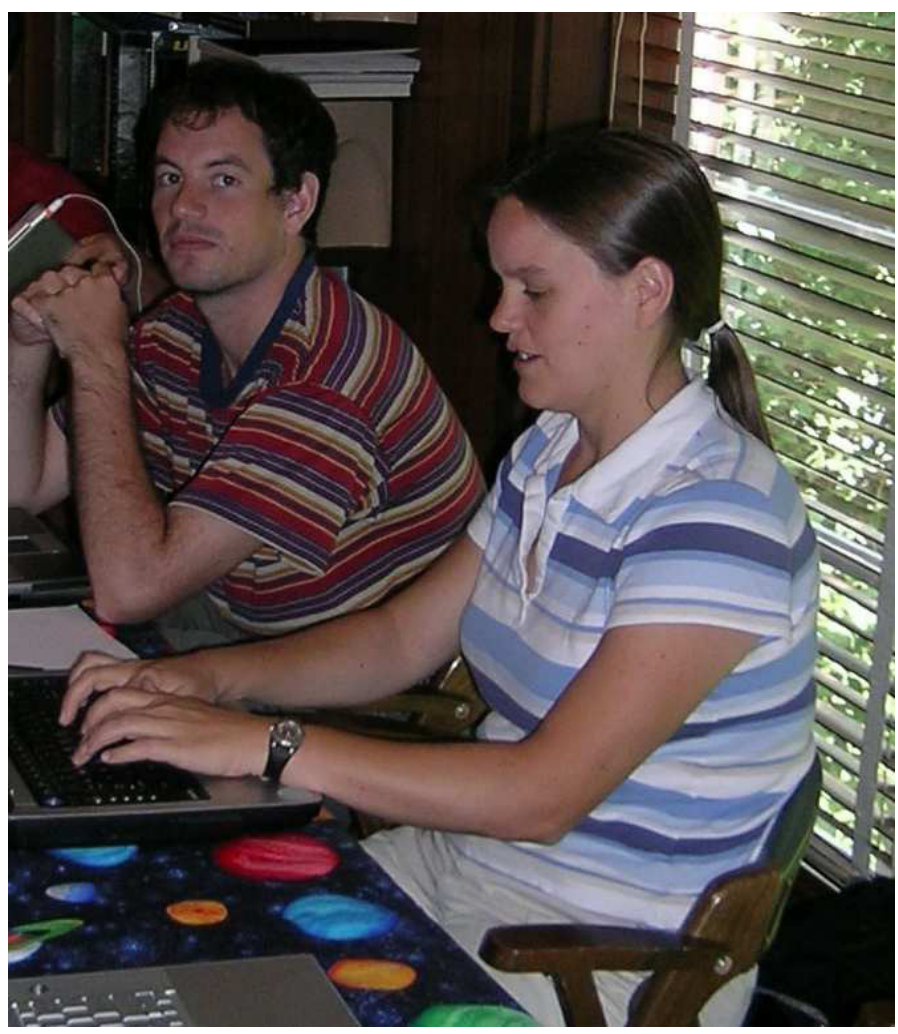

F. Mullally and S. E. Thompson taking notes in the back of the room. 